\title{
CHARACTERIZATION OF THE BEHAVIOUR OF DISSOCIATED NEURONS EXPOSED TO DIELECTROPHORETIC FORCES
}

\author{
T. Heida ${ }^{*}$ W.L.C. Rutten ${ }^{*}$, and E. Marani ${ }^{* *}$,
}

* Institute for BioMedical Technology, Faculty of Electrical Engineering University of

Twente, P.O.Box 217, 7500 AE Enschede, The Netherlands

** Dept. of Physiology, Faculty of Medicine, University of Leiden, Postbus 9604

2300 RC Leiden, The Netherlands

E-mail: t.heida@el.utwente.nl

\begin{abstract}
The behaviour of cortical rat neurons exposed to dielectrophoretic forces is investigated by varying the amplitude and frequency of the applied field. The number of neurons trapped in the center of a planar quadrupole micro-electrode structure is determined for two different amplitudes ( $3 \mathrm{~V}$ and $5 \mathrm{~V}$ ) and six different frequencies in the range from $1 \mathrm{MHz}$ to $18 \mathrm{MHz}$. A contradictory trend is found for the yield of trapped neurons for the two amplitudes as a function of the frequency.
\end{abstract}

Keywords: Dielectrophoresis (DEP), Clausius-Mosotti factor, neuron, micro-electrode structure

\section{INTRODUCTION}

A 'cultured probe' might serve as an interface between a disconnected part of the nervous system and an external stimulation circuit. This probe consists of a cultured neuronal network on a multi micro-electrode plate. To stimulate, via this interface, the neurons should be positioned on, or in the neighbourhood of, an electrode. There are several methods for the positioning of cultured cells, like chemical, mechanical, and topographical modification of the substrate on which the cells are grown. Another method, which is shown here, is electrical manipulation by means of dielectrophoresis (DEP). Dielectrophoretic forces, the forces that are exerted on polarizable particles when they are exposed to non-uniform fields [2], can 'trap' the neurons and keep them localized. The time-averaged DEP force $\mathrm{F}_{\mathrm{DEP}}$ in an $\mathrm{AC}$ electric field is dependent on the in-phase component of the dipole moment, and can be written as

$\overrightarrow{\mathrm{F}}_{\mathrm{DEP}}(\omega)=2 \pi \mathrm{r}^{3} \varepsilon_{\mathrm{m}}^{\prime} \operatorname{Re}\left[\frac{\varepsilon_{\mathrm{p}}^{*}-\varepsilon_{\mathrm{m}}^{*}}{\varepsilon_{\mathrm{p}}^{*}+2 \varepsilon_{\mathrm{m}}^{*}}\right] \nabla \overrightarrow{\mathrm{E}}_{\mathrm{rms}}^{2}$

with the complex permittivity/conductivity $\varepsilon^{*}=\varepsilon^{\prime}-i \varepsilon^{\prime \prime}=\varepsilon^{\prime}-i \frac{\sigma^{\prime}}{\omega}=\frac{-i \sigma^{*}}{\omega} \quad(\omega=2 \pi f)$, where $f$ is the frequency of the electric field, the index $\mathrm{p}$ denotes the particle (neuron), the index $m$ denotes the medium, and the index rms denotes the rms (root-mean-square) value of the electric field. Figure 1 gives an example of an electrode configuration and a surface of constant force created by it.

\section{METHODS}

Calculation of the so-called Clausius-Mosotti factor $\left(\mathrm{f}_{\mathrm{CM}}\right)$, which is a mathematical presentation of the interaction between the electric properties (conductivity and permittivity) of the neurons as well as that of the medium in which they are suspended, gives the frequency-dependency of the dielectrophoretic force as a function of $\varepsilon_{\mathrm{p}}{ }^{*}$ and $\varepsilon_{\mathrm{m}}{ }^{*}$.

$\mathrm{f}_{\mathrm{CM}}=\frac{\varepsilon_{\mathrm{p}}^{*}-\varepsilon_{\mathrm{m}}^{*}}{\varepsilon_{\mathrm{p}}^{*}+2 \varepsilon_{\mathrm{m}}^{*}}$

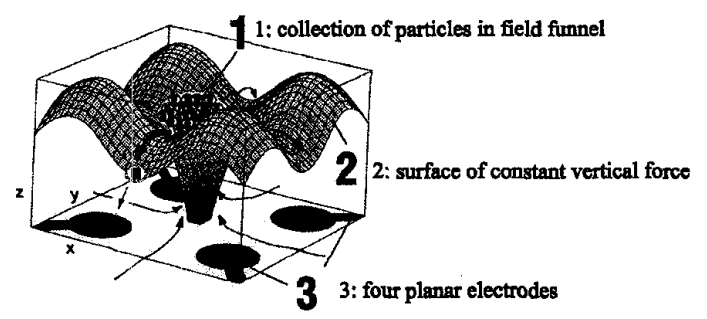

Figure 1: The principle of particle trapping in highfrequency electric fields created by a planar quadrupole electrode structure (Source: [2]).

The real part of this factor $\left(\operatorname{Re}\left[f_{\mathrm{CM}}\right]\right)$ determines the direction of the dielectrophoretic force. Due to negative dielectrophoresis (NDEP) the neurons are directed away from the electrodes in contrast to positive dielectrophoresis $(P D E P)$. For manipulating biological cells it is preferable to use negative dielectrophoretic forces in order to distance the cells from regions of high field intensities with the additional advantage that the cells are brought into stable positions. By calculating $\mathrm{f}_{\mathrm{CM}}$ as a function of the frequency, the frequency-range that is needed to direct the neurons to the regions of low field intensities (NDEP) could be determined.

Photolithographic, plasma deposition and etching techniques were used to create quadrupole and 8-pole micro-electrode structures on a glass plate of $5 \times 5 \mathrm{~cm}$. Figure 2 gives the fabrication protocol in short. A titaniumlayer was used for the adhesion of the gold-layer on the glass plate. An insulation layer, consisting of a siliconnitride layer sandwiched between two silicon-oxide layers, was applied to avoid electrochemical processes at the 
electrode-culture medium interface and to reduce unnecessary heating of the medium, since its conductivity is rather high. The 'active regions', the regions where the electric fields for dielectrophoresis are created between the electrode tips of the electrode structures, were kept free of this insulation layer.

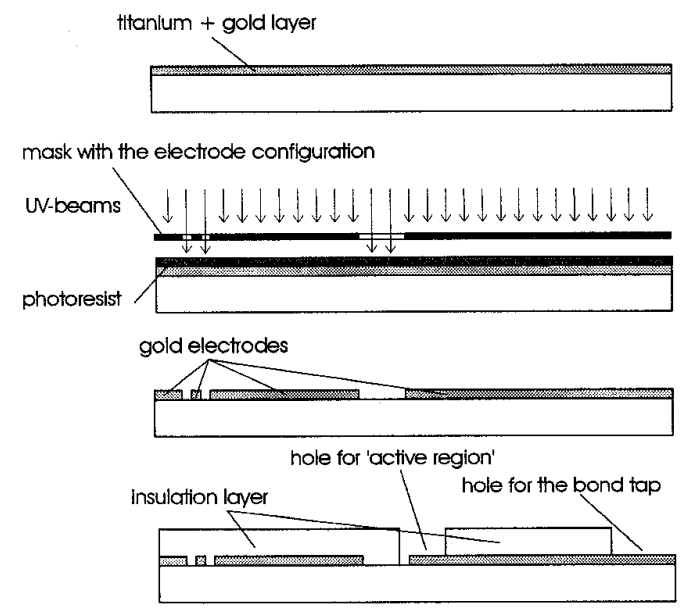

Figure 2: The fabrication protocol. After the deposition of a gold layer the electrode configurations are obtained via $U V$-radiation and etching. An insulation layer is applied over the unnecessary electrode surfaces.

Around the electrode structures a glass ring was applied which forms the 'culture chamber' (see Figure 3). The micro-electrodes were directed to two opposite sides of the electrode plate.

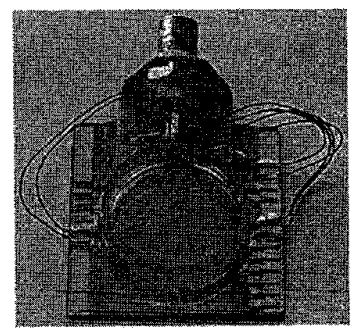

Figure 3: The electrode plate; the connector for the application of the high-frequency electric fields and the culture chamber are directly mounted onto this electrode plate.

Cortical neurons from rat fetuses (of embryonic day 18) were used for the experiments in this study. The brains were removed from the decapitated fetuses, the meninges of the cortexes were taken away and the basal ganglia as well as the hippocampus were removed. The remaining cortexes were collected in a tube with the chemical defined R12 culture medium [3]. Dissociation and centrifugation at a rate of $1400 \mathrm{rpm}$ for 5 minutes loosened the cells. Before using them for the experiments they were counted and medium was added so that a suspension of $10^{6}$ neurons $/ \mathrm{ml}$ was obtained.
A micro-electrode structure with 'triangularly shaped' electrode tips with an inter-electrode distance between two opposing electrodes of $100 \mu \mathrm{m}$ was used for the characterization of the influence of dielectrophoretic forces on dissociated cortical neurons. The small curvatures of the electrode tips provide high field gradients resulting in large dielectrophoretic forces. A cell suspension $\left(20 \mu \mathrm{l}\right.$ of $10^{6}$ cell $/ \mathrm{ml}$ ) was pipetted into the $4 \mathrm{~mm}$ ring placed around the active region while the electrodes were driven with the input signal.

At two different amplitudes of the input signal ( $3 \mathrm{~V}$ and 5 $\mathrm{V})$ in combination with six different frequencies of this signal $(1 \mathrm{MHz}, 4 \mathrm{MHz}, 8 \mathrm{MHz}, 12 \mathrm{MHz}, 14 \mathrm{MHz}, 18$ $\mathrm{MHz}$ ) the yield of cells in the center of the electrode structure was determined. This yield gives a measure of the dielectrophoretic force as a function of the amplitude and frequency of the applied field. The experiments were time lapse recorded by taking an image every 10 seconds for a time period of 30 minutes. For each setting three experiments were performed and the results of these experiments were averaged.

\section{RESULTS}

In order to calculate the Clausius-Mosotti factor a neuron was represented by a single-shell model. With this model the polarization-conduction terms from all possible, and eventually simultaneously active basic molecular mechanisms responding to the external field, are lumped together in one 'effective pair' of terms for any region of the neuron. The following parameter values were assumed for the neuron: radius $\mathrm{r}=5 \mu \mathrm{m}$, interior conductivity (cytoplasm) $\sigma_{\text {int }}=0.75 \mathrm{~S} / \mathrm{m}$, interior permittivity $\varepsilon_{\text {int }}=80^{*} \varepsilon_{0}$ (with $\varepsilon_{0}$ the permittivity of free space), membrane capacitance $c_{\mathrm{m}}=1.8 * 10^{-3} \mathrm{~F} / \mathrm{m}^{2}$, and the conductivity of the medium in which the neurons were suspended $\sigma_{\text {med }}=1.6$ $\mathrm{S} / \mathrm{m}$.

As can be seen from the calculation of $f_{C M}$ (figure 4) the entire frequency range can be used for the collection of neurons by negative dielectrophoretic forces. It is, however, recommended to use rather high frequencies ( $>100 \mathrm{kHz}$ ) [4] to avoid excessive charging of the cell membrane.

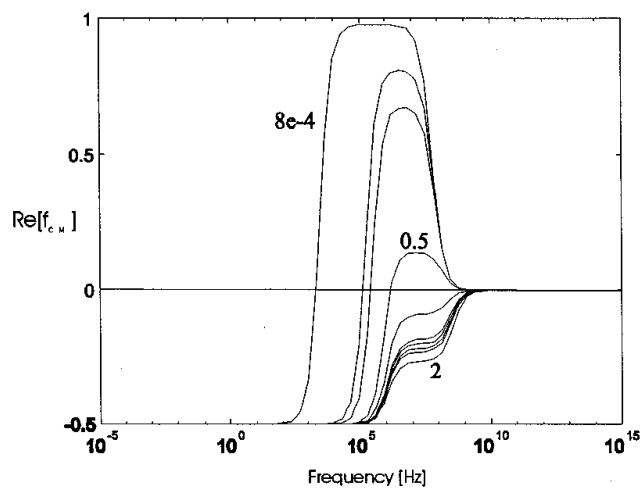

Figure 4: The real part of the Clausius-Mosotti factor for neurons as a function of the frequency using different conductivities of the medium $(8 e-4,0.05,0.1,0.5,1.4,1.5$, $1.6,1.7,2 \mathrm{~S} / \mathrm{m})$. 
Figure 4 shows that when using media of high conductivity $(>0.5 \mathrm{~S} / \mathrm{m})$ the real part of the Clausius-Mosotti factor as a function of the frequency is always negative as was already found by [5].
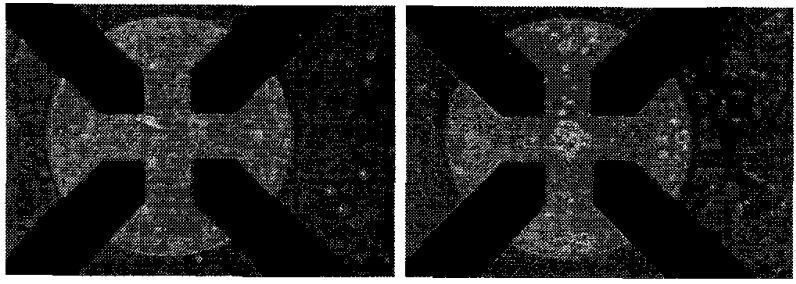

A

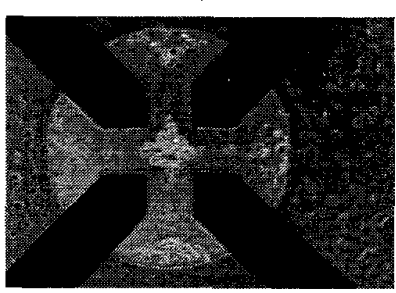

C

Figure 5: Collection of neurons by a quadrupole electrode structure $(5 \mathrm{~V}, 14 \mathrm{MHz}) \mathrm{A}$ : at the onset of the experiment $(t=0) ; B$ : after 10 minutes; $C$ : after 30 minutes. The neurons move to the center region of the four electrodes as well as to the outward regions; away from the regions of high field intensities.

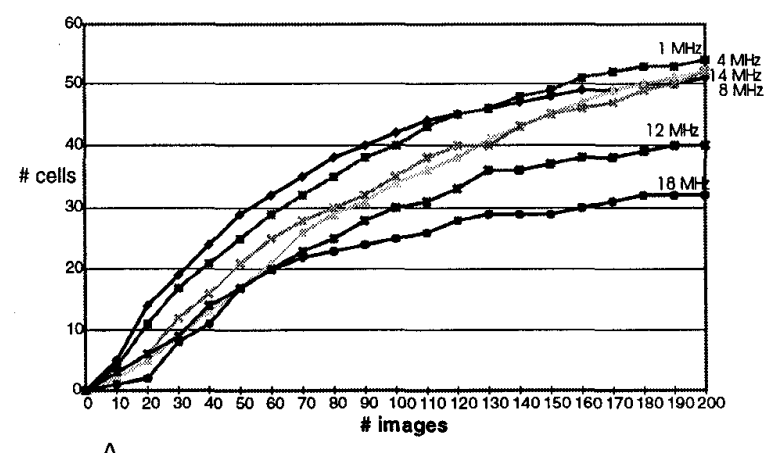

A

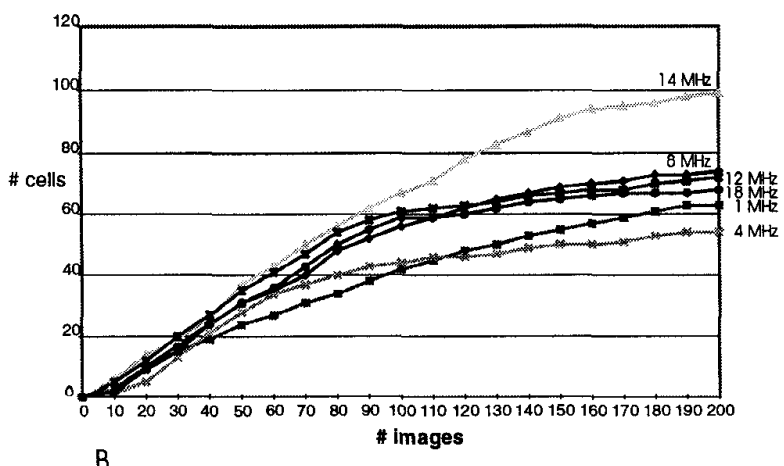

Figure 6: The number of cells trapped in the center as a function of time (\# images) at different frequencies. A: the amplitude was set at $3 \mathrm{~V}$; B: the amplitude was set at $5 \mathrm{~V}$.
Experiments showed that the cells were directed to the center of the electrode structure away from the electrodes, due to the negative dielectrophoretic forces. Figure 5 shows the situation at the start of an experiment, as well as the situation after 10 and 30 minutes.

The yield during the time span of the experiment results in the curves as shown in figure 6. Here, the number of cells trapped in the center of the electrode structure as a function of time (i.e. number of time lapse images) is shown. The curves of figure $6 \mathrm{~A}$ and $\mathrm{B}$ show that in most cases the increase in the number of cells that are trapped is largest during the first 20 minutes (120 images). After this period the number of cells that were trapped stabilizes. The standard deviation ranges from 7 to 20 after 30 minutes (180 images) of field application. During the first 10 minutes the number of cells increases linearly over time. This can also be seen in figure 7. Figure $7 \mathrm{~A}$ and $\mathrm{B}$ show the number of cells trapped up to 10 minutes at an amplitude of $3 \mathrm{~V}$ and $5 \mathrm{~V}$, respectively, using different frequencies.

With this data the yield as a function of the frequency can be investigated. This is shown in figure 7 when the field has been applied for 10 minutes at 3 and $5 \mathrm{~V}$. Nearly in all situations an amplitude of $5 \mathrm{~V}$ results in a higher number of collected cells compared to an amplitude of $3 \mathrm{~V}$.
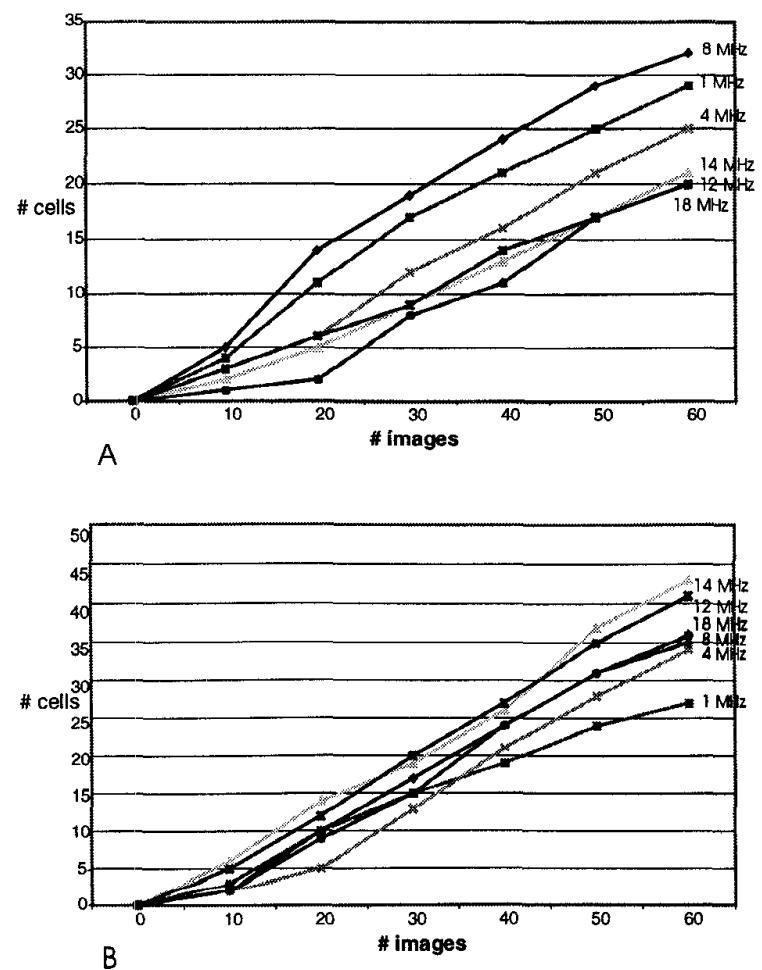

Figure 7: The number of cells trapped in the center as a function of time (\# images) during the first 10 minutes of the experiment at different frequencies. A: the amplitude was set at $3 \mathrm{~V} ; \mathrm{B}$ : the amplitude was set at $5 \mathrm{~V}$. 


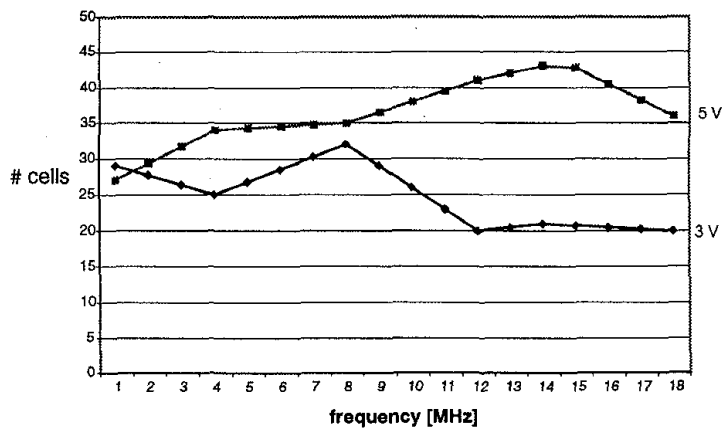

Figure 8: The number of cells trapped in the center after 10 minutes as a function of the frequency of the applied field when the amplitude is set at $3 \mathrm{~V}$ and $5 \mathrm{~V}$.

\section{DISCUSSION}

The graph of the real part of the Clausius-Mosotti factor (Figure 2), i.e., the dielectrophoretic force, suggests that the number of cells collected should be stable of slightly decrease by increasing frequency. Although a very small frequency range was investigated compared to the frequency range over which the Clausius-Mosotti factor was calculated, only for the case that an amplitude of $3 \mathrm{~V}$ is used this trend can be found. This cannot be concluded when looking at the curve showing the number of cells trapped using an amplitude of $5 \mathrm{~V}$ over this frequency range (figure 8).

To be able to characterize the dielectrophoretic force, it might be preferable to look over a shorter time scale than 30 minutes in order to avoid mechanisms that may become more apparent with time, like the heating of the medium. The curves of figure $7 \mathrm{~A}$ and $\mathrm{B}$ show that the yield of cells over a time period of 10 minutes is almost linear for all experiments. But a contradictory trend exists when comparing the number of cells trapped with the frequencies for the two amplitudes after 10 minutes of field application. Raising the frequency results in a decrease in the number of cells trapped at an amplitude of $3 \mathrm{~V}$. Using an amplitude of $5 \mathrm{~V}$ results in an increase in the number of cells trapped when the frequency is raised. Some mechanism is present that inverts the behaviour of the cells and/or the medium when the amplitude is increased. One suggestion could be that the calculation of the Clausius-Mosotti factor might not give a good representation of reality due to the incomplete knowledge of the electric properties of neurons.

It was proposed by Pohl (1986) [6] that the gradient of the square of the field varies as the square of the applied voltage. From figure 7 no similar relationship between the number of cells trapped and the amplitude can be seen. In particular, a frequency of $1 \mathrm{MHz}$ resulted in a larger number of cells trapped at $3 \mathrm{~V}$ compared to $5 \mathrm{~V}$. This may also be the result of a miscalculation of the ClausiusMosotti factor.
But, not only the dielectrophoretic force is exerted on the neurons, also viscous and gravitational forces act on the cells. Due to local heating of the medium a significant fluid flow may arise. In the center of the electrode structure an upward flow occurs and by circulation of the fluid, a flow over the surface of the electrode plate will bring the fluid back to the 'active region'. Therefore, cells that whirled down in the outward regions can be brought from these regions to the center of the electrode structure through the force of this flow. Here, they will also experience a dielectrophoretic force which might keep them trapped there. This force might contribute to the larger number of cells trapped at $5 \mathrm{~V}$ after 30 minutes, but it does not explain the contradictory trend between the two amplitudes in relation with the frequency. The possibly occurring flow forces were not yet taken into consideration.

Simulation of the electric field in three dimensions at different amplitudes and frequencies using a finite element method can help to get more insight in the dielectrophoretic force patterns. By coupling of different domains it may also be possible to simulate the fluid flow created by heating effects.

\section{ACKNOWLEDGEMENTS}

We thank Jan van Nieuwkasteele, Ton Verloop, and Ed Droog for the technical support of the fabrication of the micro-electrode plate and Marga Deenen for cortex neuron preparation.

\section{REFERENCES}

[1] H. A. Pohl "Dielectrophoresis." Cambridge: Cambridge University Press, 1978.

[2] Th. Schnelle, T. Müller, S. Fiedler, S. G. Shirley, K. Ludwig, A. Herrmann, G. Fuhr, B. Wagner, U. Zimmermann, "Trapping of viruses in high-frequency electric field cages," Naturwissenschaften, vol. 83 pp. 172 176, 1996.

[3] E. Marani, M. Corino., R. J. van den Berg, W. J. Rietveld, M. Deenen, W. Windhorst, "Ionic conductances in cultured pre-infundibular cells from the hypothalamic arcuate region," Neuroendocrinology, vol. 48 pp. $445-452$, 1988

[4] G. Fuhr, T. Müller, Th. Schnelle, R. Hagedorn, A. Voigt, S. Fiedler, "Radio-frequency microtools for particle and live cell manipulation," Naturwissenschaften, vol. 80: pp. 528-535, 1992.

[5] T. Müller, A. Gerardino, Th. Schnelle, S. G. Shirley, F. Bordoni, G. De Gasperis, R. Leoni, G. Fuhr, "Trapping of micrometre and sub-micrometre particles by high-frequency electric fields and hydrodynamic forces," The Journal of Physics, Section D: Applied Physics, vol. 29, pp. 340-349, 1996

[6] H. A. Pohl, "Theoretical aspects of dielectrophoretic deposition and separation of particle," Electrochemical society news and reviews, vol. 115, no. 6, pp. 155C-161C, 1968. 